\title{
Component Analysis in Saffron (Crocus sativus L.) for Floral and Vegetative Attributes
}

\author{
Mohammad Irfan, Sabina Nasseer, Gowhar Ali, Sabiya Bashir, Zahida Rashid, \\ Shabeena Majeed*, Shabir H. Wani, Faisal Rasool, S. Shafiq Hakeem and Z. A. Dar
}

Division of Plant Breeding and Genetics, DARS, Rangreth, India

*Corresponding author

Keywords

Crocus sativus L., Cluster, Correlation, Divergence, Genetic, Variability

Article Info

Accepted:

07 October 2020

Available Online:

10 November 2020

\section{A B S T R A C T}

To generate information on the phenotypic and genotypic variability for morphological attributes one hundred and forty (140) saffron (Crocus sativus L.) germplasm lines were evaluated for component analysis at Saffron Research Station Pampore, SKUASTKashmir during 2017-18. Observations were recorded on 10 randomly selected and tagged competitive plants for 18 morphological traits. Traits were analyzed by employing Principal Component Analysis (PCA). Combined PC1 and PC2 accounted for $68.527 \%$ of the total variance of data. Both PC1 and PC2 were characterized by nine variables showing their contribution and correlation between variables and factors. In PC1 this is appropriate regarding that Pearson correlation showed significant correlation between these variables, however the variables style length and stigma length show negative relationship with each other. The PC2 is characterized by positive relationship of stigma length and style length. In PC1 the variables, viz, leaf length (LFL), number of leaves corm $^{-1} \operatorname{line}^{-1}$ (LCL), number of flowers corm ${ }^{-1}$ line $^{-1}$, total flower weight corm $^{-1}$ (FWT/C), big corm index (BCI), fresh pistil weight (FPW) show maximum contribution towards yield.

\section{Introduction}

Saffron (Crocus sativus L.) belongs to family Iridaceae, plants of this family are herbs with rhizomes, corms or bulbs. Saffron is a perennial herbaceous plant attaining a height of 25 to $40 \mathrm{~cm}$ having sub hysteranthous behaviour. Foliar structure and floral organs constitute main parts of saffron plants (Nehvi and Salwee, 2010). Biological cycle is completed in 5 to 6 stages. It has two year plant cycle which starts from the month of July of the first year and the apical buds take a year to acquire its maximum size, and becomes a fully grown corm, while it takes another year before it is depleted and ends up with a wrinkled black disc. Usually the degradation of mother corm is visible at the beginning of November which looks quite wrinkled and flat (Medina, 2003). The species in the genus Crocus have underground fleshy corms, dark green leaves with whitish median strip. Their leaves appear with or after flowers. A plant may have one or several flowers. 
Saffron is currently being cultivated more or less intensively in Iran, India, Greece, Spain, Italy, Turkey, France, Switzerland, Israel, Pakistan, Afghanistan and recently Australia (Tasmania) (Nehvi et al., 2006). Jammu and Kashmir, the second largest contributor of saffron to the global market, is famous for its quality saffron production, high in chemical constituents like crocin, picro-crocin and saffranal. These major components of saffron are believed to characterize saffron as an anticancer medicinal herb (Abdullah, 2002), lowering blood pressure (Soeda, et al., 2001) and is used for wounds, fractures and joint pain etc. Crocin is responsible for the colour of saffron, where as picro-crocin and saffranal are responsible for its bitter taste and aroma (De Juan et al., 2009). Therefore, efforts were made to identify cultivars with high yielding potential in order to increase the production of saffron. A major step towards achieving this goal of increasing productivity of saffron, through specific plant breeding programme are to be initiated, thereby improving its cultivation. Therefore there is a need to investigate the variability in genetic material to initiate a systematic focused breeding programme.

\section{Materials and Methods}

Saffron corms weighing $5 \mathrm{~g}$ to $16 \mathrm{~g}$ were planted under each category supplemented with adequate nutrients as per the recommendations of SKUAST-K, Shalimar. The material for study comprised of 140 saffron germplasm collected from different saffron growing areas of Kashmir and abroad. The pedigree details of all the 140 corm samples was recorded and subsequently planted in Augmented Block Design with a row length of $3 \mathrm{~m}$, width $2 \mathrm{~m}$ and inter and intra-row spacing of 20 and $10 \mathrm{~cm}$, respectively. Observations were recorded on 10 randomly selected and tagged, competitive plants from each line for all the traits during the crop year 2017-2018. The observations were recorded for all the morphological traits.

Floral attributes: Number of flowers corm1line-1, Number of flowers corm-1 line-1, Total flower weight corm-1, Outer tepal length, Inner tepal length $(\mathrm{cm})$, Outer tepal width $(\mathrm{cm})$, Inner tepal width $(\mathrm{cm})$, Anther length $(\mathrm{cm})$, Anther width $(\mathrm{mm})$, Style length (cm), Stigma length $(\mathrm{cm})$, Fresh pistil weight per line (mg), Dry pistil weight per line (mg), Leaf length $(\mathrm{cm})$, No. of leaves per line.

\section{Results and Discussion}

One hundred and forty (140) saffron germplasm were evaluated for component analysis for diversity analysis of indigenous and imported saffron. Regarding the relationship between saffron yield attributing traits, these traits were analysed by employing Principal Component Analysis (PCA) as presented in Table 1 and 2. Combined PC1 and PC2 accounted for $68.527 \%$ of the total variance of data. Both PC1 and PC2 were characterized by nine variables showing the contribution of variables to the correlation between variables and factors. In PC1 this is appropriate regarding that Pearson correlation show significant correlation between these variables, however the variables style length and stigma length show negative relationship with each other. Also it has been observed that there is no correlation between style length and leaf length.

The PC2 is characterized by positive relationship of stigma length and style length. The Pearson correlation show negative relationship with rest of yield attributing traits. In PC1 the variables viz, leaf length (LFL), number of leaves corm-1line-1 (LCL), number of flowers line-1, total flower weight corm-1 (FWT/C), fresh pistil weight (FPW) show maximum contribution towards yield and also these variables have strong 
correlation with the other factors also. The nine variables had distributed scores all around the centre indicating a large amount of variability. The results show that only two factors that have Eigen values greater than one are retained or selected. These two factors constitute the variables which show best results regarding their contribution towards yield and correlation between variables and factors.

Principal component analysis was done for traits scored in field. The number of principal components calculated from correlation matrix is nine which is similar to number of observed traits. PCA concentrated variability in first two principal components under field condition; total variance explained with the first two PC's was $68.527 \%$. In the principal components where the values of yield attributing traits (Eigen values) was reduced to less than unity, which in present study occurred after the second principal component together accounting for more than $68.527 \%$ of total variance in the present experimental material, the rest of the components were not considered.

Yield attributing traits (Eigen values) for significant PCs ranged from 5.080 (PC1) to 1.087 (PC2). The first two PC's that were used for constructing biplot graphs explained $68.527 \%$ of total variation with first PC accounting for $56.448 \%$ mainly contributed by fresh pistil weight (FPW) (17.165\%) followed by number of flowers line-1 $(\mathrm{F} / \mathrm{L})$ $(16.766 \%)$, total flower weight corm-1 (FWT/C) (16.555\%), leaf length (LFL) $(11.119 \%)$ and number of leaves corm-1line-1 (L/C/L) (10.524\%). The second component explained $12.079 \%$ variability contributed largely by style length (STYL) (69.693\%), followed by stigma length (STGL) (20.961\%). Fresh pistil weight (FPW), number of flowers corm-1 line-1 (F/L), total flower weight corm-1 (FWT/C), leaf length (LFL) and number of leaves corm-1 line-1
(L/C/L) were strongly correlated with PC1 while as style length (STYL) and stigma length (STGL) were strongly correlated with PC2. However number of leaves corm-1 line1 (L/C/L), fresh pistil weight (FPW), number of flowers line-1 $(\mathrm{F} / \mathrm{L})$, total flower weight corm-1 (FWT/C), and dry pistil weight (DPW) were negatively correlated with PC2. PC1 and PC2 captured $68.527 \%$ of total variation indicating the trait vectors and the location of the genotypes according to their PC scores.

To ensure better visualization of trait vectors and objects and representation of the contribution of variables in explaining variability of the PCs, two separate graphs have been shown in (Fig. $1 \& 2$ ). The vertex genotypes are SSR/SD-62, SSR/SD-140, SSR/SD-67, SSR/SD-13， SSR/SD-36 and SSR/SD-63 having extreme trait responses. SSR/SD-62 had highest value for style length (7.35), SSR/SD-140 had highest value for dry pistil weight (976.25) and stigma length (4.13), SSR/SD-67 had highest value for fresh pistil weight (1026.49), SSR/SD-13 had highest value for leaf length (33.15), SSR/SD36 had highest value for $50 \%$ sprouting while as SSR/SD-63 had highest value for anther length (2.37). The genotypes that contribute most towards the traits (traits which are strongly related to vertex genotypes) are given as:

Genotypes related to stigma length are SSR/SD-41, SSR/SD-126, SSR/SD-57, SSR/SD-80, SSR/SD-89, SSR/SD-90, SSR/SD-75, SSR/SD-136 and SSR/SD-96.

Genotypes towards the trait leaf length are SSR/SD-125, SSR/SD-70, SSR/SD-74, SSR/SD-111, SSR/SD-103, SSR/SD-51 and SSR/SD-76.

Genotypes contributing towards style length (STYL) are SSR/SD-28, SSR/SD-94, SSR/SD-58, SSR/SD-112， SSR/SD-110, 
SSR/SD-100, SSR/SD-139 and SSR/SD-23. Genotypes related towards dry pistil weight (DPW) are SSR/SD-69, SSR/SD-32, SSR/ SD-52，SSR/SD-7, SSR/SD-84, SSR/SD-2, SSR/SD-29, SSR/SD-9, SSR/SD-4 and SSR/ SD-2 and fresh pistil weight (FPW) are SSR/SD-128, SSR/SD-127, SSR/SD-2, SSR/ SD-117, SSR/SD-66, SSR/SD-1, SSR/SD-43, SSR/SD-48, SSR/SD -95, SSR/SD-45, SSR/ SD-12, SSR/SD-55, SSR/SD-35, SSR/SD-59, SSR/SD-13, SSR/ SD-47, SSR/SD-53, SSR/ SD-51, SSR/SD-3, SSR/SD-72, SSR/SD-54, SSR/SD-119, SSR/ SD-101， SSR/SD-64, SSR/SD-20, SSR/SD-87.

In the present study, six variables including fresh pistil weight (FPW), number of flowers line-1 $(\mathrm{F} / \mathrm{L})$, total flower weight corm-1 (FWT/C), and leaf length (LFL) contributed above the expected average to the variability in PC1 and PC2. And also with the help of biplot technique, the polygon view of the biplot helps identify genotypes with the highest values for one or more traits. The vertex genotypes in biplot can be characterized for specific attributes, while those that are near to origin of biplot can be considered as genotypes characterized for a wide range of attributes, these results are in conformity with the results of Yan and Tinker 2006.

Table.1 Principal Component Analysis for yield attributing traits of saffron (Crocus sativus L.) Eigen values

\begin{tabular}{|l|c|c|c|c|c|c|c|c|}
\hline Eigen value & F1 & F2 & F3 & F4 & F5 & F6 & F7 & F8 \\
\hline & 5.08 & 1.09 & 0.76 & 0.67 & 0.47 & 0.43 & 0.10 & 0.08 \\
\hline Variability (\%) & 56.45 & 12.08 & 8.50 & 7.45 & 5.20 & 4.78 & 1.14 & 0.88 \\
\hline $\begin{array}{l}\text { Cumulative } \\
(\mathbf{\%})\end{array}$ & 56.45 & 68.53 & 77.02 & 84.47 & 89.68 & 94.45 & 99.12 & 100 \\
\hline
\end{tabular}

F1= STGL (Stigma length), F2= STYL (Style length), F3= LFL (Leaf length), F4= L/C/L (Number of leaves per corm per line), F5= F/L (Number of flowers per line), F6= FWT/C (Total flower weight), F7= FPW (Fresh pistil weight), F8= DPW (Dry pistil weight)

Table.2 Correlation and contribution of different traits towards PC1 and PC2

\begin{tabular}{|c|c|c|c|c|}
\hline Trait & \multicolumn{2}{|c|}{ PC1 } & \multicolumn{2}{|c|}{ PC2 } \\
\hline & Contribution & Correlation & Contribution & Correlation \\
\hline STGL & 5.047 & 0.506 & 20.961 & 0.477 \\
STYL & 0.456 & 0.152 & 69.693 & 0.870 \\
LFL & 11.119 & 0.752 & 2.296 & 0.158 \\
L/C/L & 10.524 & 0.731 & 0.134 & -0.038 \\
F/L & 16.766 & 0.923 & 1.479 & -0.127 \\
FWT/C & 16.555 & 0.917 & 1.669 & -0.135 \\
FPW & 17.165 & 0.934 & 2.031 & -0.149 \\
DPW & 8.638 & 0.662 & 1.734 & -0.137 \\
\hline
\end{tabular}




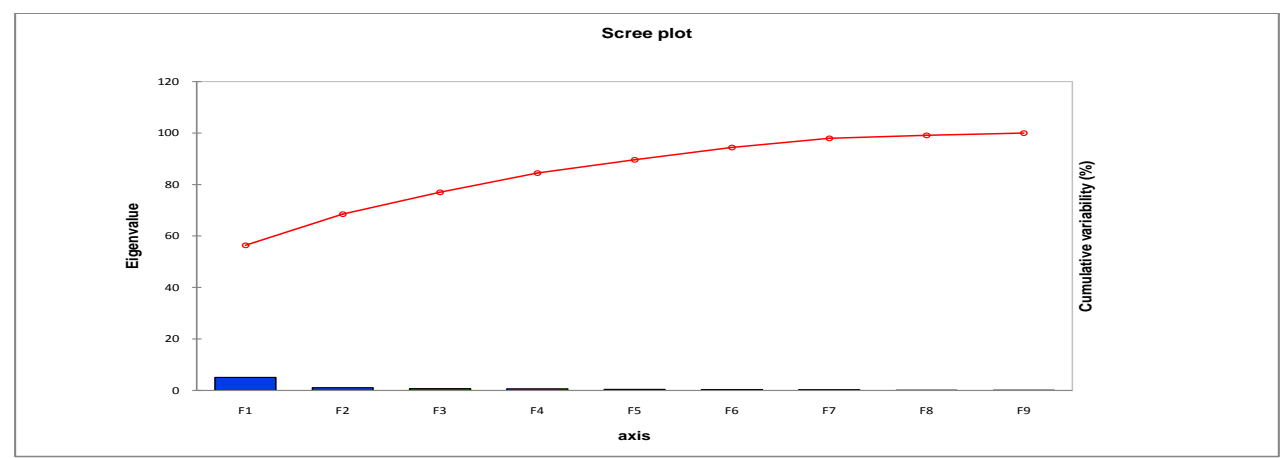

Fig.No.1. The Scree plot shows eigenvalues on $\mathrm{Y}$ axis and number of factors on $\mathrm{X}$ axis. The point where the slope of the curve is leveling off (elbow) indicates the number of factors that should be generated by the analysis. It is a simple line segment that shows the fraction of total variance in the data as explained by principal component analysis.

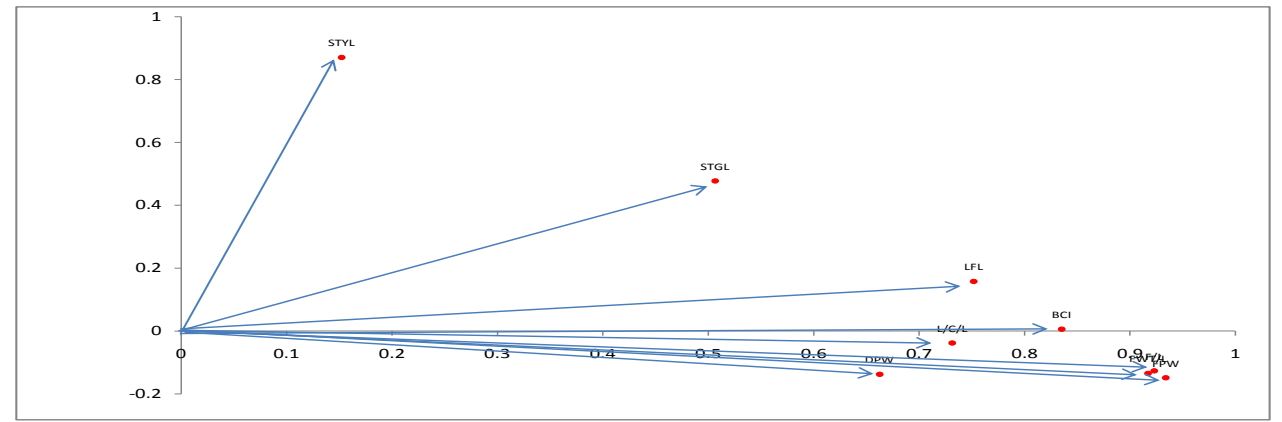

Fig. No. 2. GT biplot technique indicates the interrelationship among the variables and visual comparison among genotypes on the basis of multiple traits. It indicates that more the distance between the variables lesser they are correlated with each other. Also the angle between them indicates the inter-relationship among themselves.

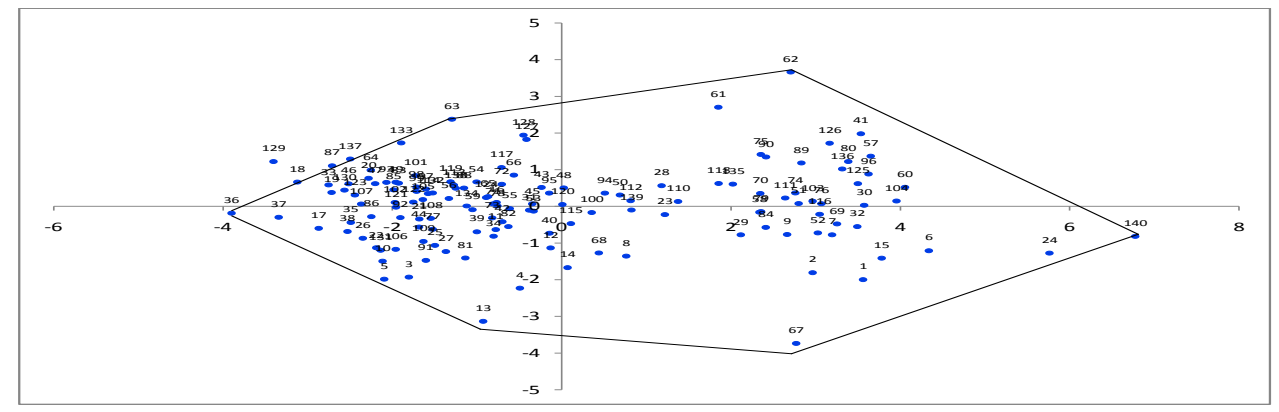

Fig.No.3. The points which are joined together are extreme genotypes and are called Vertex genotypes. Vertex genotypes; SSR/SD-62, SSR/SD-140, SSR/SD-13, SSR/SD-36, SSR/SD-63, SSR/SD-67

GT biplot graphically describes the interrelationships among all measured traits on the basis of overall pattern of the data for different genotypes and traits, whereas simple correlation coefficients only describe the relationships between two traits. Therefore, simple correlation between traits does not agree with those of relation between traits in GT biplot completely. Genotype by Trait (GT) biplot also provides a tool for visual comparison among genotypes on the basis of multiple traits. Despite being located on the vertex of the polygon, they are not always the best answer. If they are located on the left side of the biplot, these genotypes show the worst values, and care should be taken not to have an erroneous interpretation as shown in figures 1, 2 and 3.

\section{References}

Abdullah, F. (2002). Cancer chemopreventive and tumoricidal properties of saffron (Crocus sativus L.) Experimental 
Biology and Medicine227: 20-25.

De Juan, J. A., Corcoles, H. L., Munoz, R. M. and Picornell, M. R. (2009). Yield and yield components of saffron under different cropping systems. India Crop Production30: 212-219.

Medina, P. L. (2003). Anatomia e histologia de la planta de azafran (Crocus sativus L.) a lo largo de su ciclo agronomico annual. Trabajo fin de carrera IOngeniero Agronomo, Uaniversidad Castilla-La Mancha, Albacete, Espana.

Nehvi, F. A. and Salwee, Y. (2010). Saffron Farming in India The Kashmir Connection. Financing Agriculture 42:9-15.

Nehvi, F. A., Wani, S. A., Dar, S. A., Makhdoomi, M. I., Allie, B. A. and Mir, Z. A. (2006). New Emerging Trends on Production Technology of
Saffron. Proceedings of the Second International Symposium on Saffron Biology and Biotechnology28-30 October 2006. Islamic Republic of Iran, pp. 401-408.

Soeda, S., Ochiai, T., Paopong, L., Tanaka, H., Shoyama, Y. and Shimeno, H. (2001). Crocin suppresses tumpor necrosis facror alpha- induced cell death of neuronally differentiated PC12 cells. Life science 69(24); 2887-98

Yan, W. and Rajcan, I. (2002). Biplot analysis of test sites and trait relations in soyabean in Ontario Crop Science42: 11-20.

Yan, W. and Tinker, N. A. (2006). Biplot analysis of multi-environment trial data. Principles and applications. Journal of Plant sciences 86: 623-645.

\section{How to cite this article:}

Mohammad Irfan, Sabina Nasseer, Gowhar Ali, Sabiya Bashir, Zahida Rashid, Shabeena Majeed, Shabir H. Wani, Faisal Rasool, S. Shafiq Hakeem and Dar, Z. A. 2020. Component Analysis in Saffron (Crocus sativus L.) for Floral and Vegetative Attributes. Int.J.Curr.Microbiol.App.Sci. 9(11): 556-561. doi: https://doi.org/10.20546/ijcmas.2020.911.068 